\title{
Association of SLC38A4 and system A with abnormal fetal birth weight
}

\author{
ZHEN LI ${ }^{1}$, GUANGRUI LAI ${ }^{2}$, LIJUN DENG ${ }^{1}$, YUE HAN ${ }^{1}$, DANFENG ZHENG ${ }^{1}$ and WEIWEI SONG ${ }^{1}$ \\ Departments of ${ }^{1}$ Gynaecology and Obstetrics, and ${ }^{2}$ Clinical Genetics, \\ Shengjing Hospital of China Medical University, Shenyang, Liaoning, P.R. China
}

Received September 7, 2011; Accepted November 16, 2011

DOI: $10.3892 /$ etm.2011.392

\begin{abstract}
In this study, we aimed to explore the correlation between solute carrier family 38 member 4 (SLC38A4) and system A activity in human placentas from pregnancies with abnormal fetal birth weight. We collected placentas from consenting women immediately after their full-term babies were born, with normal, low birth weight or macrosomia, and used real-time PCR and Western blot analysis to detect the levels of SLC38A4 mRNA and protein [also known as sodiumcoupled neutral amino acid transport protein 4 (SNAT4)]. Isotope incorporation assay was applied to measure system A activity in the placentas. Compared to the normal birth weight (NBW) group, placentas from the fetal macrosomia (FM) group had significantly increased levels of SLC38A4 mRNA and SNAT4 (both were increased by almost 2-fold; $\mathrm{P}<0.05$ ), while no significant changes were detected in the placentas from the low birth weight (LBW) group. In addition, system A activity in the placentas from the FM and LBW groups was significantly different from that in the NBW group $(1.2 \pm 0.20$, $0.6 \pm 0.14$ vs. $1.0 \pm 0.18, \mathrm{P}<0.05)$. The data suggest that SNAT4 and system A have a strong association with abnormal fetal birth weight and that they may play a crucial role in fetal growth and development.
\end{abstract}

\section{Introduction}

Birth weight (BW) refers to the body weight within $1 \mathrm{~h}$ of birth. Full-term newborns can be divided into three categories based on their BW: i) Low birth weight (LBW), with a BW

Correspondence to: Professor Weiwei Song, Department of Gynaecology and Obstetrics, Shengjing Hospital of China Medical University, No. 36 San Hao Street, Shenyang, Liaoning 110004, P.R. China

E-mail: songww@sj-hospital.org

Abbreviations: SLC38A4, solute carrier family 38 member 4; SNAT4, sodium-coupled neutral amino acid transport protein 4; BW, birth weight; LBW, low birth weight; NBW, normal birth weight; FM, fetal macrosomia

Key words: birth weight, solute carrier family 38 member 4, sodium-coupled neutral amino acid transport protein 4 , system A of $<2,500 \mathrm{~g}$; ii) normal birth weight (NBW), with a BW of 2,500-4,000 g; and iii) fetal macrosomia (FM) with a BW of $\geq 4,000 \mathrm{~g}$. A number of factors, including pregnancy nutrition, various growth factors, hormones, and the regulatory role of the placenta, affect BW through various mechanisms, leading to abnormal fetal BW (1). Over the years, a number of investigators have found that abnormal BW is closely related to the pathogenesis of numerous adult diseases (2-4). For example, LBW and FM may lead to adult metabolic syndrome (obesity, hypertension and diabetes) through various mechanisms (5). Therefore, it is necessary to clarify the manner in which fetuses with abnormal BW differ from those with normal BW and to explain the mechanisms involved.

Currently, studies on abnormal BW are focusing on nutrition during pregnancy, a variety of growth factors, hormones, and the regulatory role of the placenta (1). It is worth noting that there are genes expressed in the placenta that control resource utilization (6). These genes affect placental function and thus play an important role in fetal growth and development (7).

Human solute carrier family 38 member 4 (SLC38A4) gene is located on chromosome $12 \mathrm{q} 13.11$. The encoded protein is known as the sodium-coupled neutral amino acid transporter 4 (SNAT4), which is a neutral amino acid transporter and a subtype of the amino acid transport system (known as system A, including SNAT1, SNAT2 and SNAT4) (8). SNAT4 is a neutral amino acid transporter in the placenta that plays a crucial role in fetal growth and development (9). It has been reported that SNAT4 is highly expressed in human placenta at the early and late stages of development (9). As an important subtype of system A, SNAT4 up-regulation leads to enhanced system A activity. In animal studies, it has been found that the inhibition of system A activity decreases the body weight of the rat fetus $(10,11)$. It has been suggested that SNAT4 and system A may be closely related to fetal development. However, to our knowledge, no such studies have been carried out in humans to date. Therefore, we studied the expression of SLC 38A4 mRNA and SNAT4 levels as well as system A activity in the human placenta in order to investigate the relevant mechanisms for abnormal fetal BW.

\section{Materials and methods}

Clinical information and specimen collection. Maternal placental tissues were collected from delivery patients in the 
Table I. Fetal birth weight (BW) and clinical information.

\begin{tabular}{lcccc}
\hline Group & Case $(\mathrm{n})$ & Fetal BW $(\mathrm{g})$ & Maternal age (years) & Gestational age (weeks) \\
\hline FM & 20 & $4,097.1 \pm 69.21^{\mathrm{a}}$ & $28.6 \pm 1.72$ & $38.8 \pm 1.16$ \\
NBW & 22 & $3,287.3 \pm 258.94$ & $29.5 \pm 2.07$ & $39.5 \pm 1.04$ \\
LBW & 10 & $2,433.3 \pm 60.72^{\mathrm{a}}$ & $27.7 \pm 1.97$ & $38.0 \pm 0.48$ \\
\hline
\end{tabular}

FM, fetal macrosomia; NBW, normal birth weight; LBW, low birth weight. ${ }^{a} \mathrm{P}<0.05$ vs. NBW.

Department of Obstetrics of Shenging Hospital of China Medical University from July 2009 to March 2010. The collections were approved by the Ethics Committee of the Shengjing Hospital, and written informed consent was obtained from all patients prior to the study. The maternal age was 25-35 years of age, gestational age was 38-42 weeks, maternal height was $155-170 \mathrm{~cm}$, father height was $165-185 \mathrm{~cm}$, all were primipara with $12.5-20 \mathrm{~kg}$ of maternal weight gain during pregnancy and without intercurrent diseases and complications, including hypertension, diabetes, heart diseases, other medical and surgical diseases, and abnormal factors of placenta and umbilical cord. The categorization of BW was carried out according to the guidelines outlined in 'Gynecology and Obstetrics'. The placentas were divided into three groups according to the fetal $\mathrm{BW}$ : i) LBW, $\mathrm{BW}<2,500 \mathrm{~g}, 10$ cases; ii) NBW, BW 2,500-4,000 g, 22 cases; iii) FM, BW $\geq 4,000$ g, 20 cases. Two sheets of placental trophoblast tissue from the central area were collected under sterile conditions. One tissue block was flash-frozen in liquid nitrogen, then stored at $-80^{\circ} \mathrm{C}$ until RNA and protein extraction. The other placental tissue block was placed in DMEM/Tyrode's (1:3) culture medium for isolation and culture of placental villous fragments to measure system A activity.

Real-time PCR to measure SLC38A4 mRNA. Total RNA was extracted using the RNA extraction kit and converted into cDNA using the cDNA reverse transcription kit (Takara, China). Real-time PCR was performed on the ABI 7500 System (Applied Biosystems, CA, USA) in a $20-\mu 1$ SYBR-Green PCR reaction containing $1 \mathrm{X}$ SYBR-Green PCR master mix (Takara), $10 \mathrm{ng}$ cDNA and $100 \mathrm{nM}$ forward and reverse primers synthesized specific to SLC38A4 (5'-GAAATTCCAAATACC CTGCCCT-3' and 5'-GCGGTGGGTGTAATCCATCA-3') and GAPDH (5'-GCACCGTCAAGGCTGAGAAC-3' and 5'-TGG TGAAGACGCCAGTGGA-3'). The reaction condition was $95^{\circ} \mathrm{C}$ for $10 \mathrm{~min}$, followed by 40 cycles of $95^{\circ} \mathrm{C}$ for $15 \mathrm{sec}, 60^{\circ} \mathrm{C}$ for $1 \mathrm{~min}$ and $72^{\circ} \mathrm{C}$ for $30 \mathrm{sec}$. Dissociation curves were generated to ensure that a single and specific product was amplified. Cycle threshold values $(\mathrm{Ct})$ were analyzed using SDS2.0 software (Applied Biosystems), and relative quantification of SLC38A4 expression was determined using the comparative $\mathrm{Ct}$ method with the GAPDH transcript as the internal control. All experiments were repeated three times.

Western blot analysis to detect SNAT4 expression. Total protein was extracted from placental tissues and the concentration was determined using the Bradford method. Denatured protein was separated by electrophoresis and transferred onto PVDF membranes (Millipore, USA). Membranes were subsequently incubated with anti-SNAT4 antibody (Santa, USA) and GAPDH antibody (Shanghai Kangcheng, China) as the primary antibody at $4^{\circ} \mathrm{C}$ overnight, and followed by horseradish peroxidase-labeled secondary antibody (Zhongshan, China) at room temperature for $2 \mathrm{~h}$. Luminescent assay was carried out on an ECL instrument (Gene Co., China).

Isotope incorporation method to detect system A activity. System A activity was detected by ${ }^{3} \mathrm{H}-$ Proline uptake. Placenta tissues were immersed in DMEM/Tyrode's (1:3) culture medium with/without $\mathrm{Na}^{+}$at $37^{\circ} \mathrm{C}$ for $3 \mathrm{~h}$. Tissues with similar 17-estradiol (based on radioimmunoassay) concentrations were selected for subsequent assays, with the addition of ${ }^{3} \mathrm{H}$-Proline $(10.1 \mathrm{nmol} / \mathrm{ml} ; 5.1 \mathrm{Ci} / \mathrm{ml}$; PerkinElmer, USA $)+$ ATP + Tyrode's medium with/without $\mathrm{Na}^{+}$, at $37^{\circ} \mathrm{C}$ for $2 \mathrm{~h}$. The mixture was then rotated in ice water for $10 \mathrm{~min}$ before adding $1 \mathrm{ml}$ normal saline, washed three times, placed in $37^{\circ} \mathrm{C}$ distilled water for $12 \mathrm{~h}$ and transferred into $0.3 \mathrm{~mol} / 1 \mathrm{NaOH}$ for $24 \mathrm{~h}$. System A activity was measured using liquid scintillation assay.

Statistical analysis. SPSS 17.0 software was applied to analyze the data. Data are shown as the means \pm SD. One-way analysis of variance or the Student's unpaired two-tailed test were used for statistical analysis. $\mathrm{P}<0.05$ was considered statistically significant.

\section{Results}

Fetal BW, maternal age and gestational age. As listed in Table I, there were no significant differences in maternal and gestational age among the FM, LBW and NBW groups $(\mathrm{P}>0.05)$. The BW of the fetuses in the FM, LBW and NBW groups had statistically significant differences (4097.1 \pm 69.21 , $2433.3 \pm 60.72$ vs. $3287.3 \pm 258.94 ; \mathrm{P}<0.05$ ).

Placental SLC38A4 mRNA expression. As shown in Fig. 1, the $S L C 38 A 4$ mRNA expression in the placentas from the FM group was significantly different from that in the placentas from the NBW group ( $2.1 \pm 0.51$ vs. $1.0 \pm 0.30 ; \mathrm{P}<0.05)$. However there was no significant difference in placental SLC38A4 mRNA expression between the LBW and NBW group $(0.9 \pm 0.24$ vs. $1.0 \pm 0.30 ; \mathrm{P}>0.05$ )

Placental SNAT4 expression. Western blot analysis and densitometry scanning showed that placental SNAT4 expression in the FM group was significantly higher compared to 


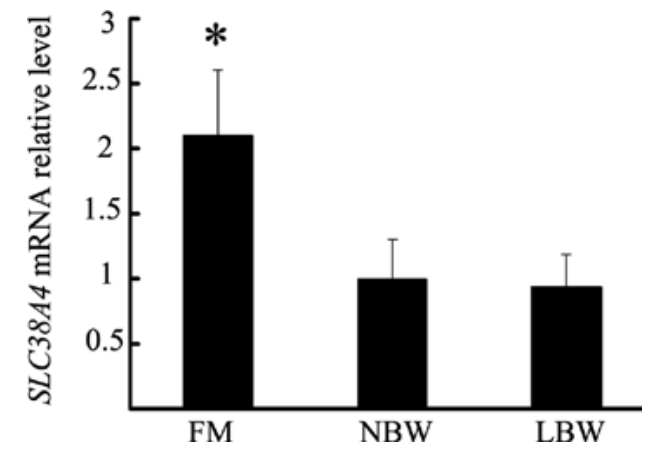

Figure 1. Placental SLC38A4 mRNA expression. FM, fetal macrosomia; NBW, normal birth weight; LBW, low birth weight. ${ }^{*} \mathrm{P}<0.05$ vs. NBW.

A

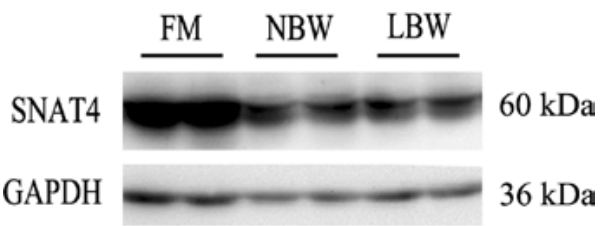

B

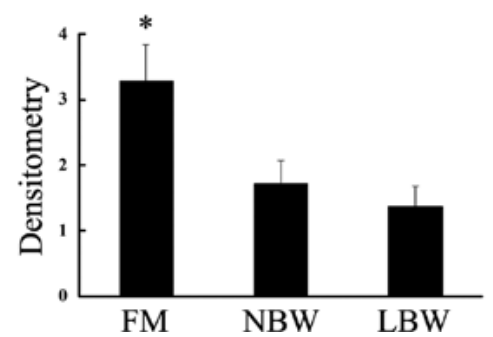

Figure 2. (A) Placental SNAT4 expression; (B) scanning densitometry of (A). FM, fetal macrosomia; NBW, normal birth weight; LBW, low birth weight. " $\mathrm{P}<0.05$ vs. NBW.

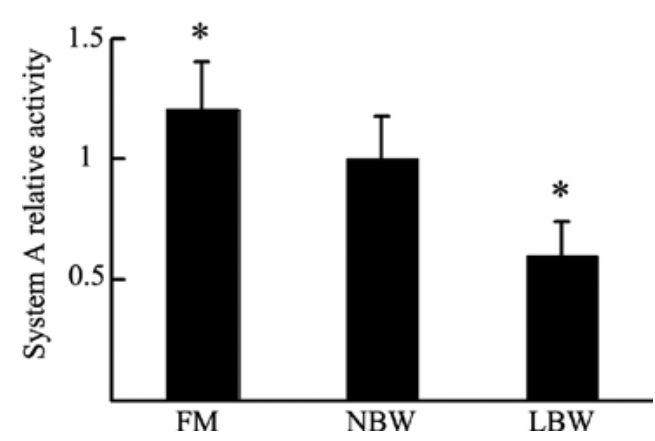

Figure 3. Placental system A activity. FM, fetal macrosomia; NBW, normal birth weight; LBW, low birth weight. "P<0.05 vs. NBW.

the NBW group. However, there was no significant difference between the LBW and NBW groups (Fig. 2).

Placental system A activity. To further explore the difference among the three groups, we used the isotope incorporation method to detect system A activity. As shown in Fig. 3, placental system A activity in the FM group was increased $(1.2 \pm 0.20$ vs. $1.0 \pm 0.18 ; \mathrm{P}<0.05)$, while that in the LBW group was decreased $(0.6 \pm 0.14$ vs. $1.0 \pm 0.18 ; \mathrm{P}<0.05)$ compared to the NBW group.

\section{Discussion}

In this study, we first found that the levels of SLC38A4 mRNA and SNAT4 in the placentas from the FM group were much higher than those in placentas from the NBW group. However, there was no significant difference in placental SLC38A4 mRNA and SNAT4 expression between the LBW and NBW groups. In addition, compared to the NBW group, placental system A activity in the FM group was increased and that in the LBW group was decreased. These results suggest that SLC $38 A 4$ and system A have a strong association with fetal BW and that they may play a key role in fetal growth and development.

Over the years, a large number of clinical studies have shown that fetuses with abnormal BW are more susceptible to adult diseases (fetal programming) $(4,5,12)$. Currently, it is believed that the factors that affect fetal BW mainly include maternal factors, nutrition and placental factors $(1,13)$. However, the placental factors may play an even greater role in BW, since placenta is the only bridge between the fetus and the extracellular matrix. The underlying mechanism could be of great clinical significance for both mother and fetus. Studies on abnormal fetal BW are now focusing on gene levels $(14,15)$, as did our current study.

The rodent $S L C 38 A 4$ gene has been proven to be a motherderived imprinted gene which promotes cell proliferation and differentiation $(16,17)$. The human SLC38A4 gene is located on chromosome 12q13.11. The protein encoded by SLC38A4 gene is known as SNAT4, a crucial component of system A, which mainly transports neutral amino acid and consists of three subtypes: SNAT1, SNAT2 and SNAT4 (formerly known as ATA1, ATA2 and ATA3, respectively), which are encoded by three members of the SLC38A gene family (SLC38A1, $S L C 38 A 2$ and $S L C 38 A 4$, respectively) $(8,18)$. These three subtypes are expressed in both rat (15) and human placental tissue $(9,20,21)$. SLC38A4 is highly expressed in the liver and was once believed to be a liver-specific gene. However, in 2006 Desforges et al found that $S L C 38 A 4$ was also expressed in human placental tissue (9). Our results confirm that SLC38A4 is expressed in the human placenta. Desforges et al also found that the expression of SNAT4 differed at various stages of pregnancy, suggesting that the SLC38A4 gene plays a diverse role at the different stages of pregnancy (22). This study focused mainly on a particular stage of pregnancy, i.e., late pregnancy, when fetal growth and development have matured and the effects of genes, proteins and other factors have stabilized, a stage that was more favorable for the aims of our study. We found that SNAT4 was highly expressed in the placentas from the FM group, which suggests that it may play a crucial role in fetal development. Similar studies have confirmed that SNAT4 is closely related to fetal growth and development in rodents (7). The mechanism involved may be that the overexpressed SNAT4 causes neutral amino acids to overload in the placenta, which promotes endometrial cell proliferation, differentiation and decidualization, enhances the invasive ability of trophoblast cells upon blastocyst implantation, and significantly affects normal development of the embryo, leading to fetal macrosomia. By contrast, no difference in placental SLC 38A4 expression between the LBW and NBW groups was found. Perhaps this could be due to the involvement of other factors, or perhaps the sample size was not large enough for the 
difference to be detected. In our experiment, the SLC $38 A 4$ mRNA levels were consistent with SNAT4 expression. Namely, the regulation on transcriptional levels played an essential role. However, we still do not know through which pathway it affected fetal development. Thus, further studies are required to explore the exact mechanism.

System A, including SNAT1, SNAT2 and SNAT4, is a $\mathrm{Na}^{+}$-dependent transporter that actively transports small, zwitterionic, neutral amino acids with short, unbranched side chains, such as alanine, serine and glutamine (23). Neutral amino acids account for the majority of total amino acids, which play a crucial and are the essential nutrients in protein formation and cell growth and differentiation. Therefore, it is conceivable that the function of system $A$ is inseparable from fetal BW. In animal studies, it was found that system A is associated with abnormal fetuses and its inhibition decreases the BW of the fetuses. Reduced system A activity has been demonstrated in microvillous membrane vesicles isolated from placentas in which the fetus was growth-restricted (24). Cramer et al reported that the inhibition of system $\mathrm{A}$ in pregnant rats leads to fetal growth restriction (10). It has also been found that alterations in placental system A activity are associated with fetal growth restriction in a rodent intrauterine growth restriction model $(11,25)$. In this study, the placental system A activity was positively correlated with the BW of fetuses in the three groups, i.e., the transport activity of system A showed an increasing trend in the LBW, NBW and FM groups, suggesting that placental system A may be one of the significant factors in determining fetal BW. In addition, Constancia et al found that enhanced system A activity was partly due to up-regulation in the expression of SNAT4 (26), indicating that SNAT4 plays a crucial role in determining the strength of system A activity. In our study, the increased placental SNAT4 expression coincided with enhanced system A activity in the FM group, which may be one of the important mechanisms of macrosomia. However, in the LBW group, although system A activity was decreased, the SNAT4 expression was not. Thus, there may be other regulatory mechanisms requiring investigation in the future. Our data also indicated that SNAT4 was not responsible for the whole system A activity, as the role of the other two subtypes (SNAT1 and SNAT2) cannot be ignored. One aspect of this study was that we could only detect system A activity, but not SNAT4 activity, to find its effect on fetal BW.

In this study, we used placentas from consenting women collected immediately after their full-term babies were born. None of the women had any intercurrent diseases and complications. However, clinical studies have shown that in several pathological pregnancy states, such as gestational hypertension and gestational diabetes (27), the frequency of fetuses with abnormal BW is greatly increased. Under these conditions, the growth conditions of the fetuses have been changed, and the demand for amino acids and other nutrients has also changed. Therefore, in our future studies we plan to investigate any changes in the amino acid transport activity at pathological pregnancy states.

LBW and FM may lead to adult metabolic syndrome. We found that system A, especially the SNAT4 subtype, was related to abnormal fetal BW. This finding may aid in the understanding of the modulatory mechanism of BW, and could lead to further studies of fetal programming mechanisms focusing on adult metabolic syndrome. Thus, it may play a facilitative role in preventing abnormal BW and reducing the incidence of increasing and non-communicable diseases that markedly affect quality of life.

\section{Acknowledgements}

This study was supported by the Liaoning Educational Research Foundation (Grant no. LS2010164).

\section{References}

1. Rastogi S, Rastogi R, Rastogi D, Rastogi R, Singh G and Chiappelli F: Evaluating the impact of a pragmatic nutrition awareness program for expectant mothers upon birth weight of the newborn. Evid Based Complement Alternat Med 2011: 185672, 2011.

2. Jaddoe VW and Witteman JC: Hypotheses on the fetal origins of adult diseases: contributions of epidemiological studies. Eur J Epidemiol 21: 91-102, 2006.

3. Ong KK and Dunger DB: Perinatal growth failure: the road to obesity, insulin resistance and cardiovascular disease in adults Best Pract Res Clin Endocrinol Metab 16: 191-207, 2002.

4. Hochberg Z, Feil R, Constancia M, et al: Child health, developmental plasticity, and epigenetic programming. Endocr Rev 32: 159-224, 2011.

5. Oken E and Gillman MW: Fetal origins of obesity. Obes Res 11: 496-506, 2003.

6. Isles AR and Holland AJ: Imprinted genes and mother-offspring interactions. Early Hum Dev 81: 73-77, 2005.

7. Angiolini E, Fowden A, Coan P, et al: Regulation of placental efficiency for nutrient transport by imprinted genes. Placenta 27: 98-102, 2006.

8. Mackenzie B and Erickson JD: Sodium-coupled neutral amino acid (System N/A) transporters of the SLC38 gene family. Pflugers Arch 447: 784-795, 2004.

9. Desforges M, Lacey HA, Glazier JD, et al: SNAT4 isoform of system A amino acid transporter is expressed in human placenta. Am J Physiol Cell Physiol 290: 305-312, 2006.

10. Cramer S, Beveridge M, Kilberg M and Novak D: Physiological importance of system A-mediated amino acid transport to rat fetal development. Am J Physiol Cell Physiol 282: 153-160, 2002.

11. Jansson N, Pettersson J, Haafiz A, et al: Down-regulation of placental transport of amino acidsprecedes the development of intrauterine growth restriction in rats fed a low protein diet. J Physiol 576: 935-946, 2006.

12. Myatt L: Placental adaptive responses and fetal programming. J Physiol 572: 25-30, 2006.

13. Haider BA, Yakoob MY and Bhutta ZA: Effect of multiple micronutrient supplementation during pregnancy on maternal and birth outcomes. BMC Public Health 11: S19, 2011.

14. Jensen RB, Chellakooty M, Vielwerth S, et al: Intrauterine growth retardation and consequences for endocrine and cardiovascular diseases in adult life: does insulin-like growth factor-I play a role? Horm Res 60: 136-148, 2003.

15. Hiden U, Lang U and Desoye G: Fetoplacental disturbances in gestational diabetes mellitus. Gynakol Geburtshilfliche Rundsch 49: 224-229, 2009.

16. Rosario FJ, Jansson N, Kanai Y, et al: Maternal protein restriction in the rat inhibits placental insulin, mTOR, and STAT3 signaling and down-regulates placental amino acid transporters. Endocrinology 152: 1119-1129, 2011.

17. Mizuno Y, Sotomaru Y, Katsuzawa Y, et al: Asb4, Ata3, and Den are novel imprinted genes identified by high-throughput screening using RIKEN cDNA microarray. Biochem Biophys Res Commun 290: 1499-1505, 2002.

18. Hediger MA, Romero MF, Peng JB, Rolfs A, Takanaga $\mathrm{H}$ and Bruford EA: The ABCs of solute carriers: physiological, pathological and therapeutic implications of human membrane transport proteins. Pflugers Arch 447: 465-468, 2004.

19. Hay N and Sonenberg N: Upstream and downstream of mTOR. Genes Dev 18: 1926-1945, 2004.

20. Desforges M, Greenwood SL, Glazier JD, Westwood M and Sibley CP: The contribution of SNAT1 to system A amino acid transporter activity in human placental trophoblast. Biochem Biophys Res Commun 398: 130-134, 2010. 
21. Jones HN, Jansson T and Powell TL: IL-6 stimulates system A amino acid transporter activity in trophoblast cells through STAT3 and increased expression of SNAT2. Am J Physiol Cell Physiol 297: 1228-1235, 2009.

22. Desforges M, Mynett KJ,Jones RL, et al: The SNAT4 isoform of the system A amino acid transporter is functional in human placental microvillous plasma membrane. J Physiol 587: 61-72, 2009.

23. Johnson LW and Smith CH: Neutral amino acid transport systems of microvillous membrane of human placenta. Am J Physiol 254: 773-780, 1988

24. Jansson T, Ylven K, Wennergren M and Powell TL: Glucose transport and system A activity in syncytiotrophoblast microvillous and basal plasma membranes in intrauterine growth restriction. Placenta 23: 392-399, 2002.
25. Constancia M, Hemberger M, Hughes J, et al: Placental-specific IGF-II is a major modulator of placental and fetal growth. Nature 417: 945-948, 2002.

26. Constancia M, Angiolini E, Sandoivi I, et al: Adaptation of nutrient supply to fetal demand in the mouse involves interaction between the Igf 2 gene and placental transporter systems. Proc Natl Acad Sci USA 102: 19219-19224, 2005.

27. Ornoy A: Prenatal origin of obesity and their complications: Gestational diabetes, maternal overweight and the paradoxical effects of fetal growth restriction and macrosomia. Reprod Toxicol 32: 205-212, 2011. 